\title{
Genomic benchmarking studies reveal variations of the polyubiquitination domain of the PSD95 protein in Homo neanderthalensis and other primates of the Hominidae family: Possible implications in cognitive functions?
}

\author{
Michael Zuarez-Chamba ${ }^{1}$, Luis Puma ${ }^{1}$, Jorge Bermeo ${ }^{1}$, Eugenio Andrade ${ }^{1}$, Stalin A. Bermúdez-Puga ${ }^{1}$ \& Leopoldo Naranjo-Briceño ${ }^{1,2^{\star}}$
}

DOI. 10.21931/RB/2021.06.01.23

\begin{abstract}
Modern humans' unique cognitive abilities regarding Neanderthals and other primate's lineages are frequently attributed to the differences in brain size development and evolution. However, recent studies have established the critical role of genomic and genetic benchmarking in analyzing the cognitive evolution between modern humans and primates, focused mainly on searching for involved genes in neurogenesis. PSD95 protein (named PSD95p) has a key role in modulating synaptic plasticity, learning, and memory skills. Thus, the present study aimed to determine the possible variations of the PSD95 gene between modern humans, Neanderthals, and other hominid primate species using bioinformatics tools. The results showed 14 polymorphisms compared with the contemporary human PSD95 gene, of which 13 were silent mutations, and only one was a non-silent mutation at the nucleotide position 281. Despite polymorphisms found at the nucleotide sequences, the PSD95p of humans and chimpanzees are 100\% identical. Likewise, the gorilla and orangutan PSD95p are 100\% identical, although a 103-amino acid deletion characterizes them at the N-terminal end (1-103), suggesting that it behaves like a non-functional protein. Interestingly, the single nucleotide polymorphism (SNP) found at position 281 in the Neanderthal PSD95 gene leads to a change of the $\mathrm{E}^{94}$ to valine $\mathrm{V}^{94}$ in the polyubiquitination domain (PEST) and variation in the three-dimensional structure of PSD95 protein. We prompt that this structural change in the PEST domain could induce a loss of PSD95p function and, therefore, an alteration in synaptic plasticity forms such as long-term potentiation (LTP) and long-term depression (LTD). These findings open a possible hypothesis supporting the idea that humans' cognitive evolution after separating our last common ancestor with Neanderthals lineage could have been accompanied by discrete changes in the PSD95p polyubiquitination domain.
\end{abstract}

Key words: Evolution, cognitive development, intelligence, memory, ARC gene, PES.

\section{Introduction}

Since our last common ancestor's divergence with Neanderthals, the early humans' evolution process, between 430,000 to 765,000 years ago ${ }^{1-4}$, and with chimpanzees, between 4 to 7 million years ago $0^{5,6}$, has become a subject of great controversy. One of the most relevant issues within this matter is precisely the interbreeding that humans and NeanderthaIs could have after migrated from Sub-Saharan Africa in two events. The first time carried out 100,000 years $\mathrm{ago}^{4}$ and the second time 50,000 years ago $0^{3,7-10}$. Based on studies of fossil remains, it has been reported that the second interbreeding event allowed the introgression of around $6-9 \%$ of the Neanderthal genome into the early human genome $e^{4,7}$. However, due to positive selection processes, a recent Asian and European genomes analysis has shown that the percentage of genetic segments introgressed from Neanderthal to human fell $1-3 \% 3,7,11,12$.

The persistent Neanderthal genes in the genome of modern non-African humans are mainly those involved in phenotypic traits, directly influenced by the environment and other genes involved in both cognitive and skeletal development ${ }^{8,9}$. Consequently, these genes have been linked to the expansion of modern humans throughout Europe, which subsequently led to the disappearance of Neanderthals 30,000 years ago ${ }^{8,13}$. Nevertheless, it is evident that beyond a disappearance, Neanderthals were diluted in the early human genome.

On the other hand, despite the evolutionary processes in time and space between modern humans and chimpanzees, unlike Neanderthals, it has been found that their genome is 98.7\% identical ${ }^{5,6}$. Despite their high identity percentage, Likewise other hominids, chimpanzees also differ from modern humans in many morphological, behavioral, and cognitive characteristics ${ }^{5}$.

Modern humans' behavior and unique cognitive abilities concerning Neanderthals and other hominid primates have been attributed to the brain's evolution process ${ }^{5,14-18}$. Several studies have suggested that the lower development of the brain's cranial capacity and organization could be the possible cause of the Neanderthal extinction ${ }^{14,18-20}$. Based on these facts, it has been reported that the Neanderthal adaptive system was unable to survive the competitive environment with modern humans $\mathrm{s}^{20-22}$, therefore, emphasizing that modern humans had a more remarkable cognitive ability to develop adaptive strategies ${ }^{4,7}$. However, a report described that the Neanderthals and modern humans had similar brain sizes, advocating that it is wrong to infer differentiated cognitive development between these two species based on archaeological data analysis ${ }^{23}$. Recent studies reveal the importance of molecular biology and bioinformatics tools for the cognitive development investigation between modern humans and their common ancestors, which are focused mainly on the search of involved genes in neurogenesis, a mechanism associated with learning and memory developments ${ }^{6,18,24}$. Therefore, it has been suggested that the evolutionary development of the brain is a direct cause of a more significant generation of neural 
networks, hence the rounded form that has been attributed to the modern human brain compared with the elongated shape of the Neanderthal brain ${ }^{24}$.

Likewise, it has been found that the regulation of genes involved in the migration and detrital development of neurons has led to a dissimilar functional development between the neurons of humans, chimpanzees, and bonobo ${ }^{18}$. In-silico analysis done in this work are not enough to confirm that the polymorphism of the PSD95 can be associated with neural networks. However, the results open a window to the scientific community because they allow knowing and infer a possible cause in the cognitive development differences between Neanderthals and modern humans and other primates.

It is known that the learning process through memory lies in the organization of neurons and their interactions ${ }^{25}$. Memories are stored in a neuronal circuit by synapse mechanisms and specific neurons ${ }^{26}$. Synaptic plasticity or dynamic changes of neuronal connections change the neuron's chemical composition and its capacity to communicate with its neighbors. Also, that allows the generation of new functional neurons (neurogenesis), contributing even more to plasticity ${ }^{27}$. The me- chanisms involved in synaptic plasticity changes are long-term potentiation (LTP) and long-term depression (LTD), characterized by an increase and weakening of the synaptic force, respectively. Therefore, the favoring of LTP and the development of memory and learning have been associated with an increased expression of the PSD95 gene, which encodes a protein of postsynaptic density 95 (PSD95p) characteristic of an electrodense region called postsynaptic density ${ }^{28,29}$.

PSD95p is a specialized scaffold protein composed of several multiple protein interaction domains that include: i) a polyubiquitination domain (PEST), ii) three PDZ domains, iii) an SH3 domain, and iv) a guanylate kinase (GK)-like domain ${ }^{30-32}$ PSD-95p is the best-studied protein of the guanylate kinase-associated membrane family (MAGUK) ${ }^{30}$. Within this family, there are other scaffolding proteins such as SAP102, SAP97, and PSD9332. PSD95p with PSD93p can interact in postsynaptic sites to form a multimeric scaffold for grouping associated receptors, ion channels, and signaling proteins ${ }^{30,33,34}$. As shown in Figure. 1, PSD95p is the anchoring axis of a complex of postsynaptic proteins, ion channels, and NMDA receptors (N-methyl-D-aspartate) and AMPA (a-amino-3-hydroxy-5-

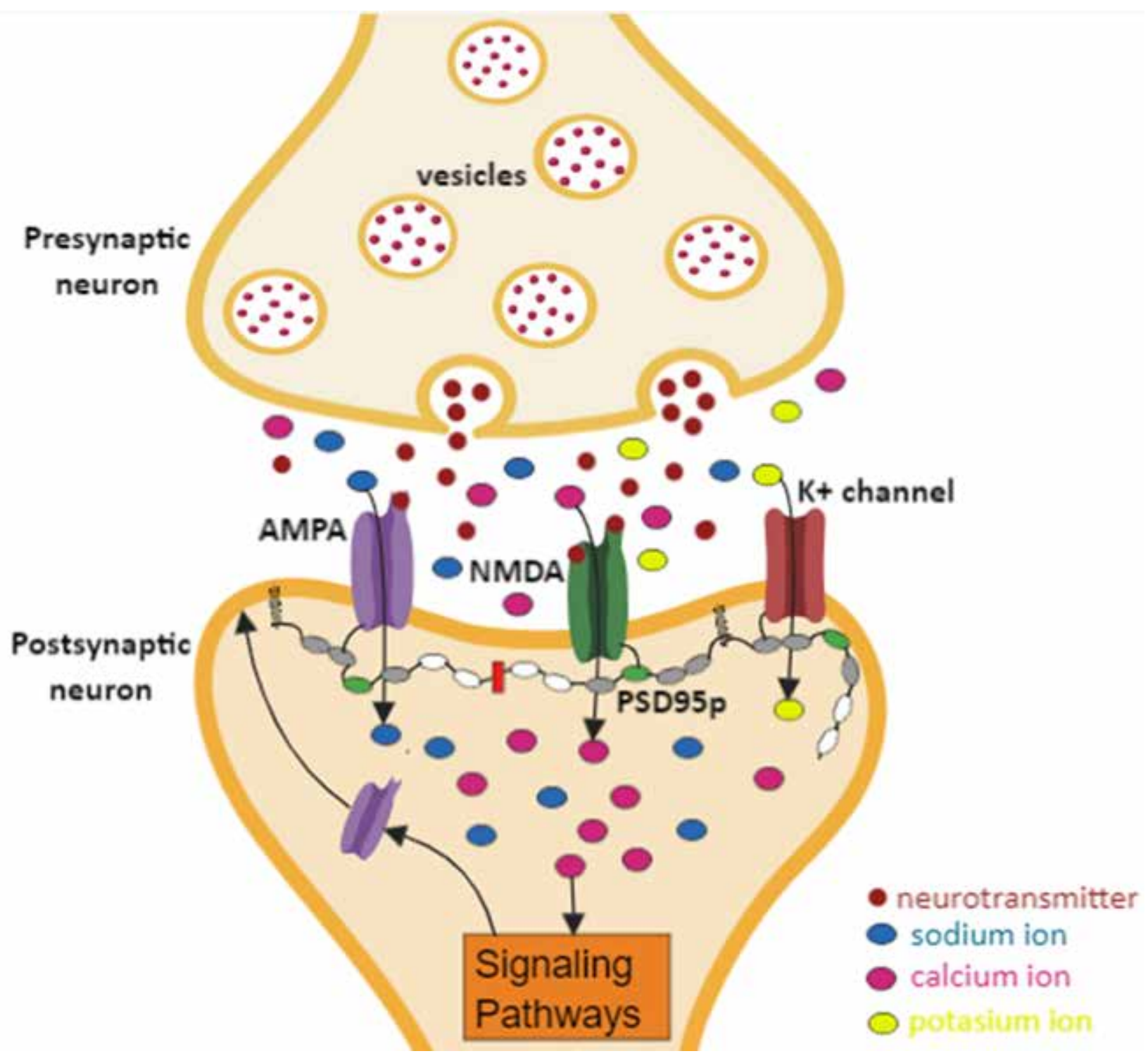

Figure 1. Interaction of PSD95p with AMPA and NMDA receptors. Generation of signaling pathways to increase the localization of AMPA receptors to the postsynaptic membrane; thus, inducing an increase of the strength of the synaptic connections between neighboring neurons. 
methyl-4-acid isoxazole propionic) type. The presence of the NMDA and AMPA proteins is essential in the synaptic plasticity and cognitive functions development. However, their correct function will depend directly on the presence of PSD95p. For this reason, this study was only focused on the function of PSD95p, which plays an essential role in synaptic plasticity during LTP by allowing better localization of AMPA receptors in the synaptic membrane; thus, inducing an increase in the synaptic connections' strength between neighboring neurons ${ }^{33-37}$.

Experiments performed in mice have shown that mutations in the gene that encodes the PSD95 protein lead to a deficit in learning and memory abilities, abnormal social behavior, repetitive behaviors, increased stress, anxiety-related responses, and different motor alterations ${ }^{34,38}$. In the case of mutations and erroneous genetic variants in the ARCp-PSD95p complex, it has been found that there may be a high susceptibility to schizophrenia, autism disorders, intellectual disabilities, epileptic mutations, and abnormal variants in intelligence ${ }^{33,39,40}$. In future works, mutation analysis in the other proteins involved with cognitive development, such as ARCp, AMPA, and NMDA receptors, among others, will be elucidated.

In this research, we hypothesized that if in an evolutionary past, humans acquired specific variations in the nucleotide sequence of the PSD95 gene, then the structure and function of PSD95p, as well as the development of cognitive abilities, could have been improved compared to Neanderthals and other primates of the Hominidae family.
(Neanderthal), Pan troglodytes (Chimpanzee), Pan paniscus (Bonobo), Gorilla gorilla (Gorilla) and Pongo abelii (Orangutan). The PSD95 nucleotide (without introns) and PSD95p amino acid sequences of human, Chimpanzee, bonobo, gorilla, and orangutan were downloaded from the Ensembl Database ${ }^{41}$ and GenBank database ${ }^{42}$. The Neanderthal's nucleotide and amino acid sequences were downloaded from the Neanderthal Genome database ${ }^{43}$, as shown in Tables 1 and 2.

Due to the present study's complexity, only one sequence was selected per Hominidae primate's species studied. However, in the case of Neanderthals, there is only one access to a sequenced genome in the Neanderthals Genome database.

In spite that this approach only uses one sequence by a specimen to make the comparison between species regarding PSD95 genes and proteins, it is useful to release scientific questions and hypothesis and to planning strong in vivo and in vitro experiments allowing to determine the relationships between variations of the polyubiquitination domain of the PSD95 protein and its possible implications in cognitive functions in Homo neanderthalensis and other primates of the Hominidae family, including modern human adequately.

\section{Prediction of 3D protein structures}

Prediction of the tertiary structure of human PSD5p was performed with Protein Homology/analogy Recognition Engine V 2.0 (PHYRE2) server ${ }^{45}$, which also allowed us to predict the 3D structure domains have not been crystallized previously. Prediction of the PSD5p 3D structures of Neanderthal, Chim-

\begin{tabular}{|c|c|c|c|c|c|c|c|}
\hline Organism & Scieatific uame & Chromosome & $\begin{array}{c}\text { Orienta } \\
\text { tion }\end{array}$ & $\begin{array}{l}\text { ID } \\
(\%)\end{array}$ & $\begin{array}{c}E \text { - } \\
\text { value }\end{array}$ & Arcession & References \\
\hline Human & Homo sapiens & $17: 7196755-7197052$ & Reverse & 100 & 0 & $\underline{\mathrm{U} 33192.1}$ & $\begin{array}{l}\text { Stathakis, Hoover, You, and } \\
\text { Bryant (1997) }\end{array}$ \\
\hline Neanderthal & $\begin{array}{l}\text { Homo } \\
\text { neanderthalensis }\end{array}$ & 17:7033933.7063745 & Reverse & 99.91 & 0 & ENSG60000132535 & $\begin{array}{l}\text { The Neanderthal Genome } \\
\text { Projest-May } 2010\end{array}$ \\
\hline Chimpanzee & Pan troglodyes & 17:7450950-7451247 & Reverse & 99.83 & 0 & $\mathrm{XM} 16931399.2$ & MODEL REFSEQ. \\
\hline Bonobo & Pan paniscus & $17: 7214437-7214734$ & Reverse & 99.48 & 0 & $\mathrm{XM} 03810105,3$ & MODEL REFSEQ. \\
\hline Gorilla & Gorilla gorilla & $17: 7202712.7203009$ & Reverse & 99.67 & 0 & $\mathrm{XM} 190131261$ & MODEL REFSEQ. \\
\hline Orangutan & Pongo abelii & $17: 7210380-7210677$ & Reverse & 99.17 & 0 & $\mathrm{XO} 2423438221$ & MODEL REFSEQ. \\
\hline
\end{tabular}

Table 1. General characteristics of the studied Hominidae primates PSD95 genes.

\begin{tabular}{|l|l|c|c|c|l|}
\hline Organism & Scientific name & ID (\%) & E- value & Accession & References \\
\hline Human & Homo sapiens & 100 & 0 & $\underline{\text { AAC52113.1 }}$ & $\begin{array}{l}\text { Stathakis, Hoover, You, } \\
\text { and Bryant (1997) }\end{array}$ \\
\hline Neanderthal & $\begin{array}{l}\text { Homo } \\
\text { neanderthalensis }\end{array}$ & 99.87 & 0 & $\underline{\underline{E N S G 00000132535}}$ & $\begin{array}{l}\text { The Neanderthal Genome } \\
\text { Project - May 2010 }\end{array}$ \\
\hline Chimpanzee & Pan troglodytes & 100 & 0 & $\underline{\mathrm{XP}_{0} 16786888.1}$ & MODEL REFSEQ. \\
\hline Bonobo & Pan paniscus & 99.61 & 0 & $\underline{\mathrm{XP}_{0} 03810153.1}$ & MODEL REFSEQ. \\
\hline Gorilla & Gorilla gorilla & 100 & 0 & $\underline{\mathrm{XP}_{0} 18868671.1}$ & MODEL REFSEQ. \\
\hline Orangutan & Pongo abelii & $\mathbf{1 0 0}$ & $\mathbf{0}$ & $\underline{\mathbf{X P}_{0} \mathbf{2 4 0 9 0 1 5 0 . 1}}$ & MODEL REFSEQ. \\
\hline
\end{tabular}

Table 2. General characteristics of the studied Hominidae primates PSD95 proteins.

The multiple sequence alignments were performed with the MUSCLE server directly linked to the Molecular Evolutionary Genetics Analysis software, MEGA X 10.0.54

\section{Materials and methods}

\section{Obtaining PSD95 sequences and multiple alignments}

The primates selected for the present study were chosen based on the species belonging to the Hominidae clade, specifically: Homo sapiens (Human), Homo neanderthalensis panzee, bonobo, gorilla, and orangutan was performed by SWISS-MODEL server ${ }^{46}$ previous model generated for humans as a template. Finally, the models obtained were analyzed using PyMOL 2.3 software ${ }^{47}$

\section{Phylogenetic trees}

Two phylogenetic trees were built using nucleotide and amino acid sequences of the PSD95 gene and the PSD95p. The 
evolutionary history was performed using MEGA $X^{48}$, the maximum likelihood method. Additionally, the Tamura-Nei model was used ${ }^{49}$ for nucleotides and the model Le_Gascuel_200850 for amino acids. To increase the reliability of the analyzed taxa's consensus trees, a bootstrap corresponding to 2500 replicates was established ${ }^{51}$.

To decrease the construction complexity of the consensus tree that presents an optimal topology, the heuristic search method was used. For this, an initial tree was automatically obtained, applying the algorithms of Neighbor-Join and BioNJ to a matrix of distances by pairs estimated using the Maximum Composition Probability (MCL) approach, for the nucleotide sequences. The heuristic search's initial tree was obtained by applying the Neighbor-Joining method to a matrix of pairwise distances estimated using a JTT model. The analysis involved 6 nucleotide sequences and 6 amino acid sequences.

\section{Results}

\section{Polymorphisms are found in the PEST domain of the PSD95 gene in Neanderthal and other primates of the Hominidae family}

Despite the high percentage of identity (Table 1), the results obtained showed 14 polymorphisms along the PSD95 gene (Figure. 2A). The Neanderthal PSD95 gene is different from the human gene only by replacing one nucleotide at position 281 ( $A^{281}$ by $T^{281}$ ). Simultaneously, the Chimpanzee is different from humans by substituting two nucleotides at positions 75 and 1503 ( $A^{75}$ by $C^{75}$ and $T^{1503}$ by $C^{1503}$ ). The bonobo PSD95 gene is discriminated from humans by the substitution of 2 nucleotides at positions 75 and 1494 ( $\mathrm{A}^{75}$ by $\mathrm{C}^{75}$ and $\mathrm{T}^{1494}$ by $\mathrm{C}^{1494}$ ) and by deletion of 9 nucleotides at positions 279-289 that are highly conserved in humans, Neanderthal, and Chimpanzee (5'...GAGTGMCAG...3'). Gorilla and orangutan were the species that presented a lower percentage of identity with humans (99.67 and 99.17, respectively). This differentiation is related to the substitution of 5 and 8 nucleotides, respectively, and the deletion of 309 nucleotides in the 5'-end at positions 1-309 concerning the human, Neanderthal, Chimpanzee PSD95 genes.

\section{Change of $A^{281}$ by $T^{281}$ in the Neanderthal PSD95 gene leads to alteration of $\mathrm{Glu}^{94}$ to $\mathrm{Val}^{94}$ in the PEST domain.}

Interestingly, as is shown in Figure 2B, the alignments of amino acid sequences showed that, from the 14 polymorphisms shown above, 13 correspond to silent mutations. Only one mutation is a non-silent type, which was found in the Neanderthal PSD95 gene. This single nucleotide polymorphism (SNP), based on substitution of adenine by thymine at position 281, led to a change of the glutamate residue at position 94 (E94R) by a valine (V94R) in the Neanderthal PSD95 protein. Similarly, the deletion of the 9 nucleotides in bonobo led to a modification of 3 amino acids (E94R-L95R-Q96R), and the deletion of 309 nucleotides in gorilla and orangutan led to a deletion of 103 amino acids at the $\mathrm{N}$-terminal end of the protein (position 1-103). On the other hand, the results indicate that, despite the polymorphisms found in the nucleotide sequences, the PSD95p protein from humans and chimpanzees are 100\% identical. Likewise, gorilla and orangutan proteins do not show specific amino acid changes. An important fact is that overall substitutions and deletions from species studied were found in the PEST domain of PSD95p, as shown in Figure 2C.
The evolutionary history based on the PSD95p protein indicates that modern humans have a greater kinship with chimpanzees than with Neanderthals.

Using the maximum likelihood method, the evolutionary lineage concerning the PSD95 gene shows us the three clades' formation; the first one groups includes human, Neanderthal, and gorilla. In this clade, we can observe that humans have a greater kinship with Neanderthals than with gorillas (Figure. 3A). The second clade includes Chimpanzee and bonobo, and the third one only consists of the orangutan.

Regarding the PSD95p protein, three clades are obtained (Figure. 3B). However, because the polymorphisms found were non-silent mutations, except for Neanderthal, differences were observed in the first two clades described above for the phylogenetic tree regarding the species' PSD95 genes studied. Thus, a first clade was observed for humans and chimpanzees, a second clade for Neanderthals, bonobo, and gorilla, where Neanderthal has a greater kinship with bonobo. Finally, no differences were observed in the third clade formed by the orangutan.

\section{Discussion}

Proteins can fold in a specific way depending on the amino acids that constitute them until forming three-dimensional structures. Consequently, the function of a protein is directly related to its structure. Based on the results based on PSD95p sequences, we have shown that chimpanzees have a higher percentage of identity with modern humans. However, studies have shown that the cognitive differences between these two species are not governed solely by structural changes in gene products as proteins and gene expression levels are involved in the different cognitive processes.

On the other hand, due to the SNP found in the Neanderthal PSD95 gene, a change of glutamate (C) by valine (V) is generated, leading to a modification in the $3 \mathrm{D}$ conformational of the protein, as shown in Figure. 4A-B. Thus, we believe that the function of the PSD95 protein from Neanderthal was possibly affected, with a concomitant reduction in cognitive function compared with modern humans.

Likewise, as a consequence of the SNP found in the Neanderthal PSD95 gene, a change of glutamate (C) by valine $(V)$ is generated, leading to a modification in the $3 \mathrm{D}$ conformational of the protein and therefore in its function (Figure. 4).

According to previous studies, a change of the glutamate amino acid within the PEST domain may lose its function. The synaptic strength depends on the AMPA and NMDA type receptors, regulated in part by the PSD95 protein, which seems depleted ${ }^{52,53}$. Then, based on the conservation of the E94RL95R-Q96R motif (Figure. 5), it could be thought that the modification in the E94R residue in Neanderthal could have induced a loss of function of the PSD95p protein. Likewise, the signal peptide's de-palmitoylation at the $\mathrm{N}$-terminal end of the protein can weaken synaptic strength and lead to an LTD. This sequence of about 24 amino acids, found upstream of the PEST domain when undergoing a palmitoylation process in cysteine, serine, or threonine residues, allows PSD95p to be coupled postsynaptic membrane for subsequent anchoring of receptors and other signaling and adhesion proteins ${ }^{36,54,55}$. Therefore, based on the mentioned above, it could be inferred that the deletion of the $\mathrm{N}$-terminal end of 103 amino acids in the gorilla and orangutan protein may be, among other elements, a probable cause that they have less cognitive development than humans. Since PSD95p doesn't have a palmitoylation 
A

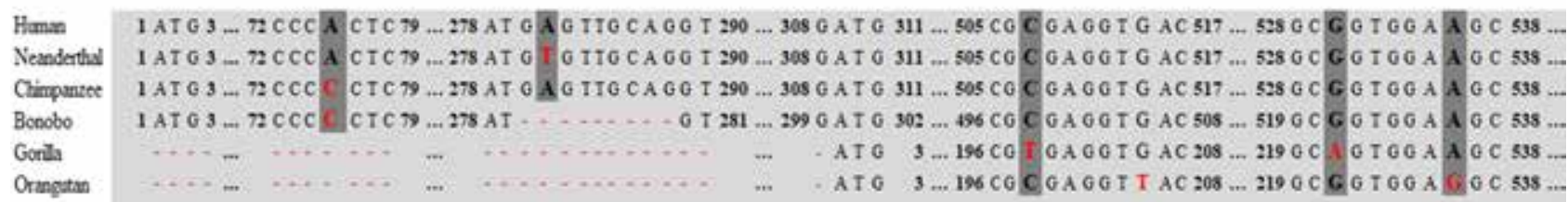

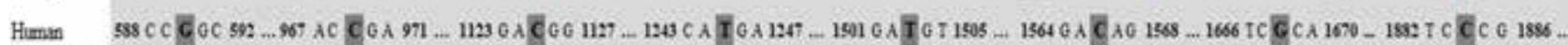

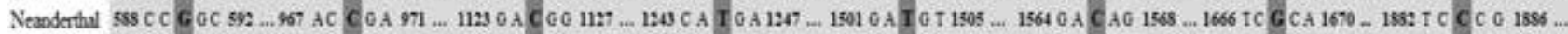

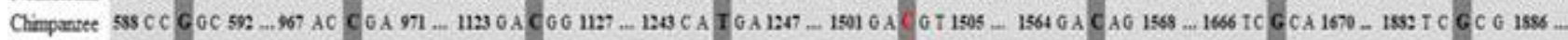

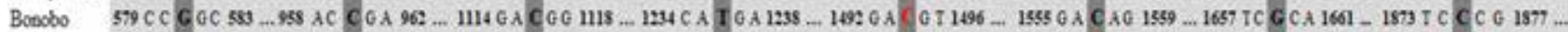

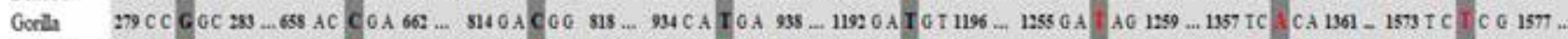

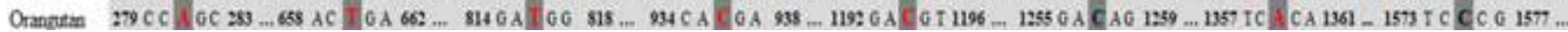
B

Human I MSQRPRAPRSALWLLAPPLLRWAPPLLTVLHSDLFQALLDILDYYEASLSESQKYRYQDE 60 Neanderthal 1 MSQRPRAPRSALWLLAPPLLR WAPPLLTVLHSDLFQALLDILDYYEASLSESQRYRYQDE 60.. Chimpanzee 1 MSQRPRAPRSALWLLAPPLLRWAPPLLTVLHSDLFQALLDLDYYEASLSESQKYRYQDE 60... Bonobo 1 MSQRPRAPRSALWLLAPPLLRWAPPLLTVLHSDLFQALLDILDYYEASLSESQKYRYQDE 60.. Gorilla Orangutan

Human 61 DTPPLEHSPAHLPNQANSPPVI VNTDTLEAPGYELQVNGTEGEMEYEETTLERGNSGLGF 120.. Neanderthal 61 DTPPLEHSPAHLPNQANSPPVI VNTDTLEAPOY LQVNGTEGEMEYEEITLERGNSGLGF 120.. Chimpanzee 61 DTPPLEHSPAHLPNQANSPPVI VNTDTLEAPGYELQVNGTEGEMEYEEITLERGNSGLGF 120... Bonobo 61 DTPPLEHSPAHLPNQANSPPVI VNTDTLEAPGY...VNGTEGEMEYEEITLERGNSGLGF 120.

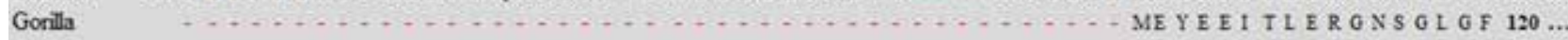

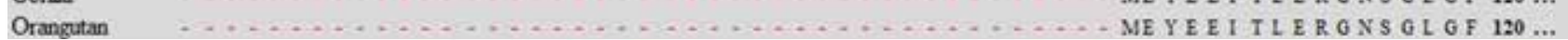

C

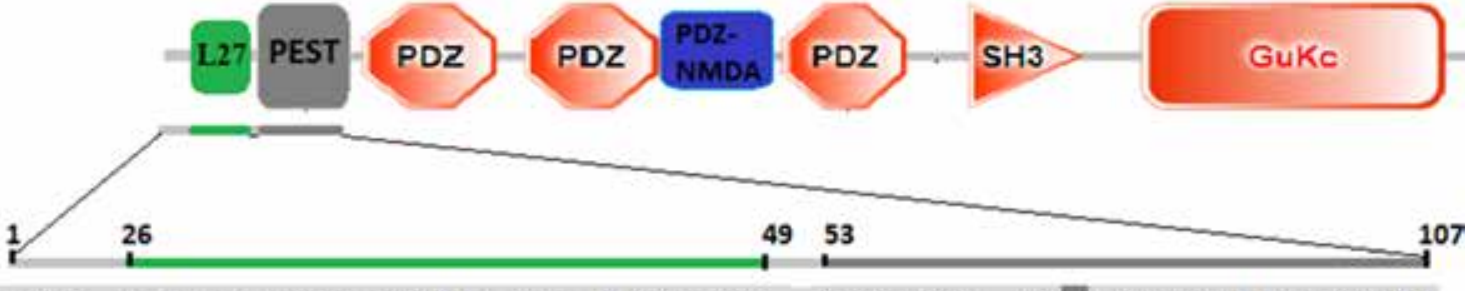

Human 1 MSQ..LLTVLHSDLFQALLDILDYYEASL_. QKYRYQ... Y YLQVNGTEGE MEYE Neanderthal 1 MS Q...LLTVLHSDLEQALLDILD Y TEASL .. QKYRYQ ...GYYLQVNGTEGEMEYE Chimpanzee 1 MSQ...LLTVLHSDLFQALLDILDYYEASL_. QKYRYQ...GYELQVNGTEGE MEYE Bonobo $\quad 1$ MSQ...LLTVLHSDLFQALLDILDYYEASL_. QKYRYQ...GY... VNGTEGEMEYE Gorilla Orangutan ME Y E color; substitution mutations are designated with red letters; deletion mutations are designated with dotted lines of red color. Multiple alignments of the amino acid sequences. (B) Amino acids that have been substituted are represented with a red-letter, deletions are designated with dotted red lines. (C) Localization of the mutations found in Neanderthal, bonobo, gorilla, and orangutan. The green stripe represents the L27 domain consisting of 24 amino acids. The Grayline represents the polyubiquitination domain (PEST) composed of 55 amino acids.

site, it cannot be located in the postsynaptic membrane for later anchoring of receptors and other proteins participating in the modulation of the synaptic force.

On the other hand, it is known that PSD95p can form supercomplexes with both NMDA and non-NMDA receptors ${ }^{37}$. These supercomplexes are the ARC protein (ARCp), which has been associated with learning processes and intelligence development. According to Fernandez et al. (2017), PSD95p is one of the proteins that interacts with ARCp in a superior percentage, finding that mutations in the PSD95p-ARCp complex can lead to a learning impairment dependent on the hippocampus $^{33}$. The exciting results obtained in the present work could give the first evidence of improved intelligence development in humans compared to Neanderthals by allowing a modulation between LTP and LTD. This is necessary to decrease the synaptic connections between neurons that do not follow a sequential pattern due to the same stimulus, at the same time, increasing the strength of synaptic connections between neurons that are correlated ${ }^{56}$

Finally, it has been established that the postsynaptic localization of AMPA receptors mediated by PSD95p plays a vital role in the regulation intracellular $\mathrm{Ca}^{+}$ion concentrations in postsynaptic cells ${ }^{57,58}$. The increase in $\mathrm{Ca}^{+}$is thought to be a generator of retrograde signaling that induces a more significant release of neurotransmitters from the presynaptic neuron to the synaptic space, leading to the same time to excitotoxicity ${ }^{59}$. Also, according to Zhang et al., (2014), the high concentrations of intracellular $\mathrm{Ca}^{+}$lead to the activation of the $\mathrm{Ca}^{+}$dependent calmodulin protein, which, when bound to the N-terminal end of PSD95p, inhibits the palmitoylation of PSD95p and, consequently, its dislocation from the postsynaptic membrane ${ }^{60}$.

As a result, higher production of NO is induced, subsequently resulting in the membrane's lipoperoxidation and destabilization of the postsynaptic receptors ${ }^{59,61}$. Consequently, 

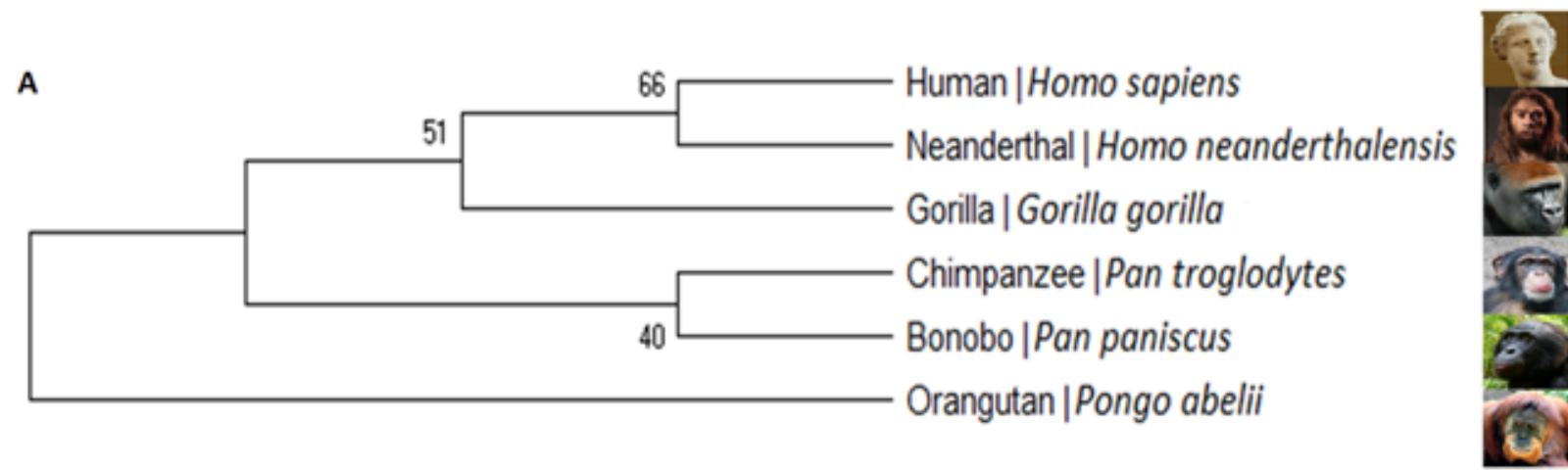

1598
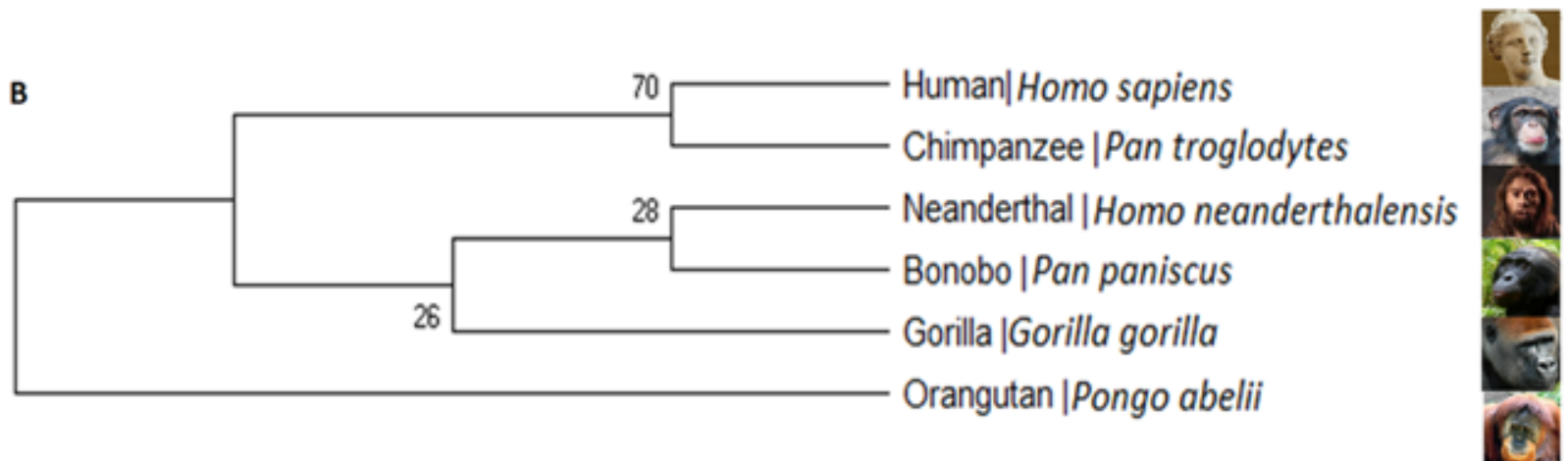

Figure 3. Evolutionary history of the Hominidae primate's family concerning the PSD95 gene and its encoded protein. (A) The genes' evolutionary history was inferred using the Maximum Likelihood method and the Tamura- Nei model. (B) The evolutionary history concerning the proteins was inferred by using the Maximum Likelihood method and Le_Gascuel_2008 model. The bootstrap consensus tree inferred from 2500 replicates both for genes and proteins represent the evolutionary history of the taxa analyzed. Branches corresponding to partitions reproduced in less than 50\% bootstrap replicates are collapsed. Percentages of replicate trees in which the associated taxa clustered together in the bootstrap test (2500 replicates) are shown next to the branches.

pathologies, such as depression, post-traumatic stress disorder, and stroke are generated ${ }^{62}$. Therefore, it is suggested that proteolysis by the proteasome of human PSD95p induced by polyubiquitination could act as a regulatory mechanism in the disengagement of AMPA receptors to decrease the flow of $\mathrm{Ca}^{+2}$ ions dependent on NMDA receptors (Figure. 6), favoring in this way the modulation of the synaptic force between neighboring neurons and so the increase of cognitive capacity.

\section{Conclusions}

In conclusion, these findings support the idea that the cognitive evolution of modern humans after the separation of our last common ancestor with Neanderthals and other hominid primates could be have been accompanied by discrete changes in the domain of polyubiquitination of PSD95p, which has a crucial role in the modulation of synaptic plasticity and cognitive development. However, it should be considered that PSD95p is not the only protein involved in synaptic plasticity processes. Therefore, to verify our claims, it is necessary to perform in vitro and in vivo experiments to evaluate that the change of glutamate by valine at position 94 in the PEST domain could lead to the loss of the PSD95p functions and concomitant deterioration of cognitive development. The in-silico findings presented in this work are only the beginning and could open a window for future robust investigations.

\section{Acknowledgment}

We thank the excellent opportunity to study at the prestigious Universidad Regional Amazónica Ikiam. The authors thank A. Naranjo, for your grammatical support.

\section{Competing Interests}

Authors declare there is no competing of interest

\section{Bibliographic references}

1. Arsuaga, J. L. et al. Neandertal roots: Cranial and chronological evidence from Sima de los Huesos. Science (80-. ). 344, 13581363 (2014).

2. Prüfer, K. et al. The complete genome sequence of a Neanderthal from the Altai Mountains. Nature 505, 43-49 (2014).

3. Enard, D. \& Petrov, D. A. Evidence that RNA Viruses Drove Adaptive Introgression between Neanderthals and Modern Humans. Cell 175, 360-371.e13 (2018).

4. Kuhlwilm, M. et al. Ancient gene flow from early modern humans into Eastern Neanderthals. Nature 530, 429-433 (2016).

5. Enard, W. et al. Intra- and interspecific variation in primate gene expression patterns. Science (80-. ). 296, 340-343 (2002).

6. Liu, X. et al. Extension of cortical synaptic development distinguishes humans from chimpanzees and macaques. Genome Res. 22, 611-622 (2012).

7. Fu, Q. et al. An early modern human from Romania with a recent Neanderthal ancestor. Nature 524, 216-219 (2015).

8. Green, R. E. et al. A draft sequence of the neandertal genome. Science (80-. ). 328, 710-722 (2010).

9. Pääbo, S. The diverse origins of the human gene pool. Nature Reviews Genetics 16, 313-314 (2015).

10. Sankararaman, S., Patterson, N., Li, H., Pääbo, S. \& Reich, D. The Date of Interbreeding between Neandertals and Modern Humans. PLoS Genet. 8, (2012). 

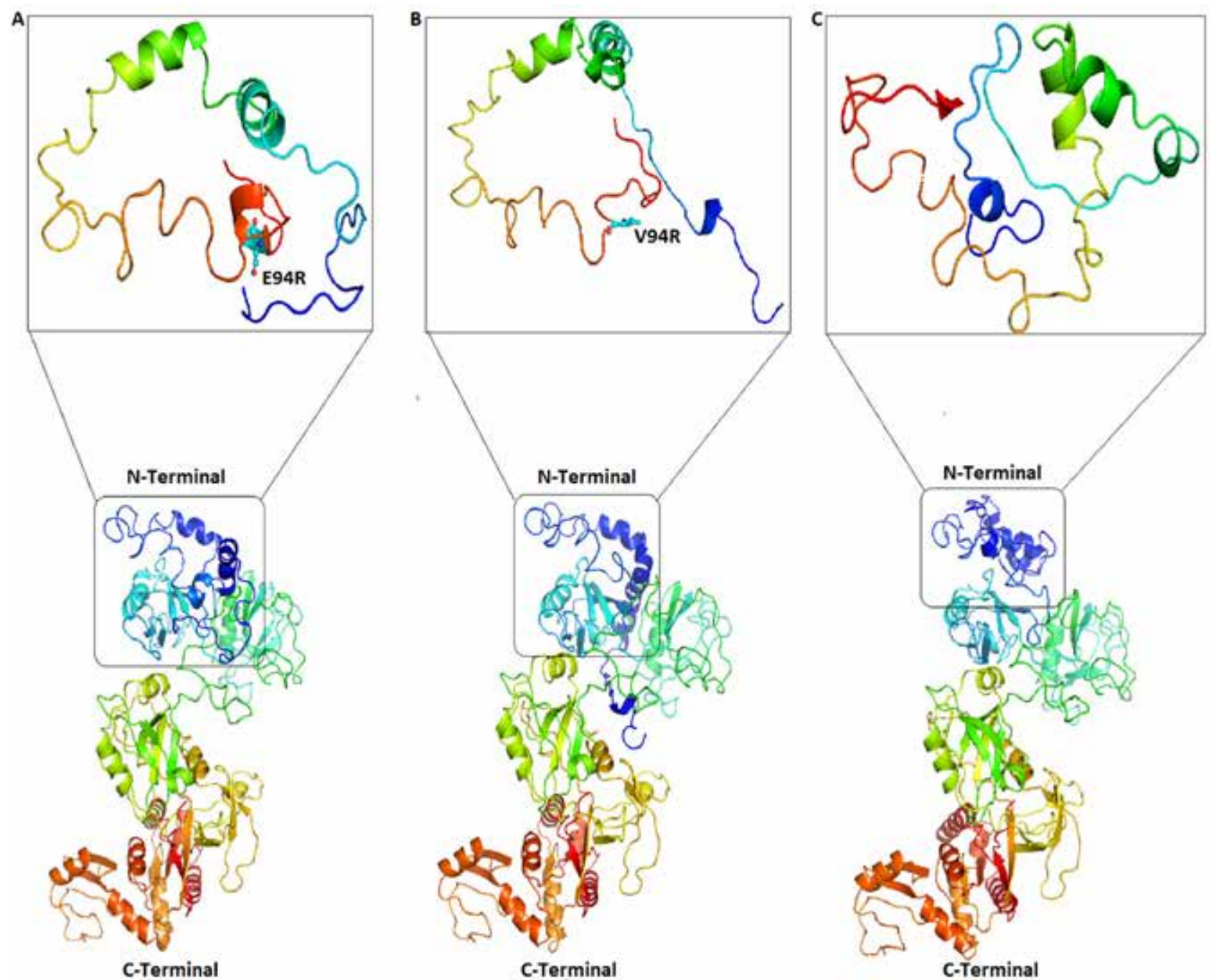

Figure 4. Conformational changes in the PSD95p three-dimensional structure. (A) Complete 3D structure prediction of human and chimpanzee proteins and the 3D structure of its $\mathrm{N}$-terminus end are shown, indicating the glutamic amino acid E94 in balls and sticks figures. (B) Prediction of the 3D structure of the Neanderthal PSD95p shows that its N-terminal end differs from human protein. (C) The bonobo protein 3D structure is indicated, and, as well as the Neanderthal's, the $\mathrm{N}$-terminal end diverges from human PSD95p.

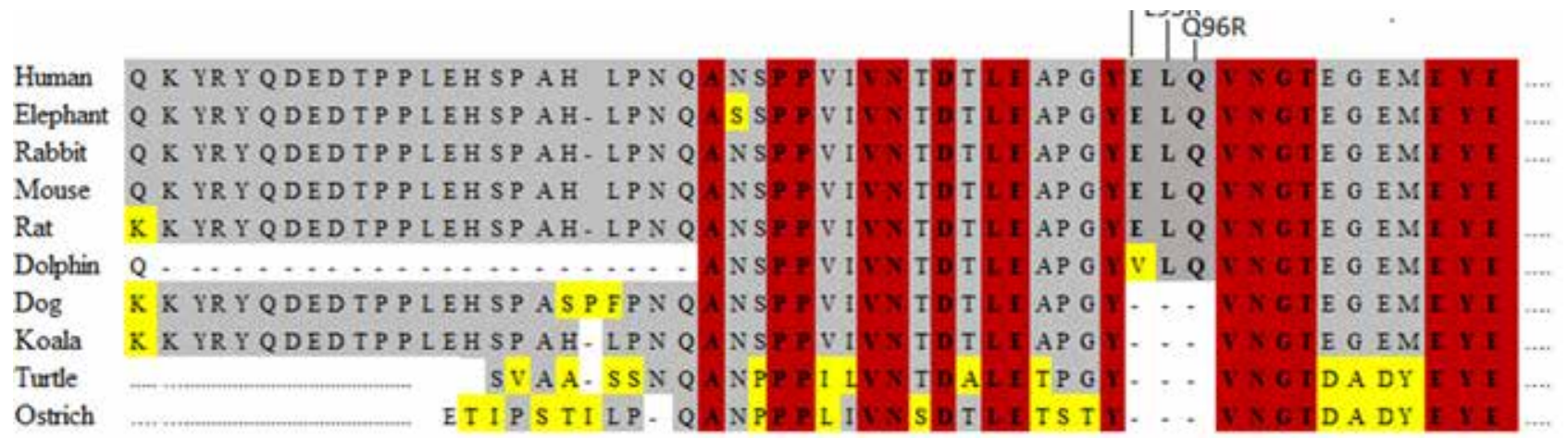

Figure 5.CConserved motifs in the PEST domain of the N-terminal end of diverse macro vertebrate proteins. The red color indicates fully conserved motifs; the gray color indicates partially conserved motifs and, the yellow color indicates the non-conserved amino acids. 


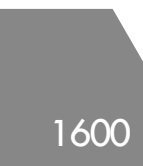

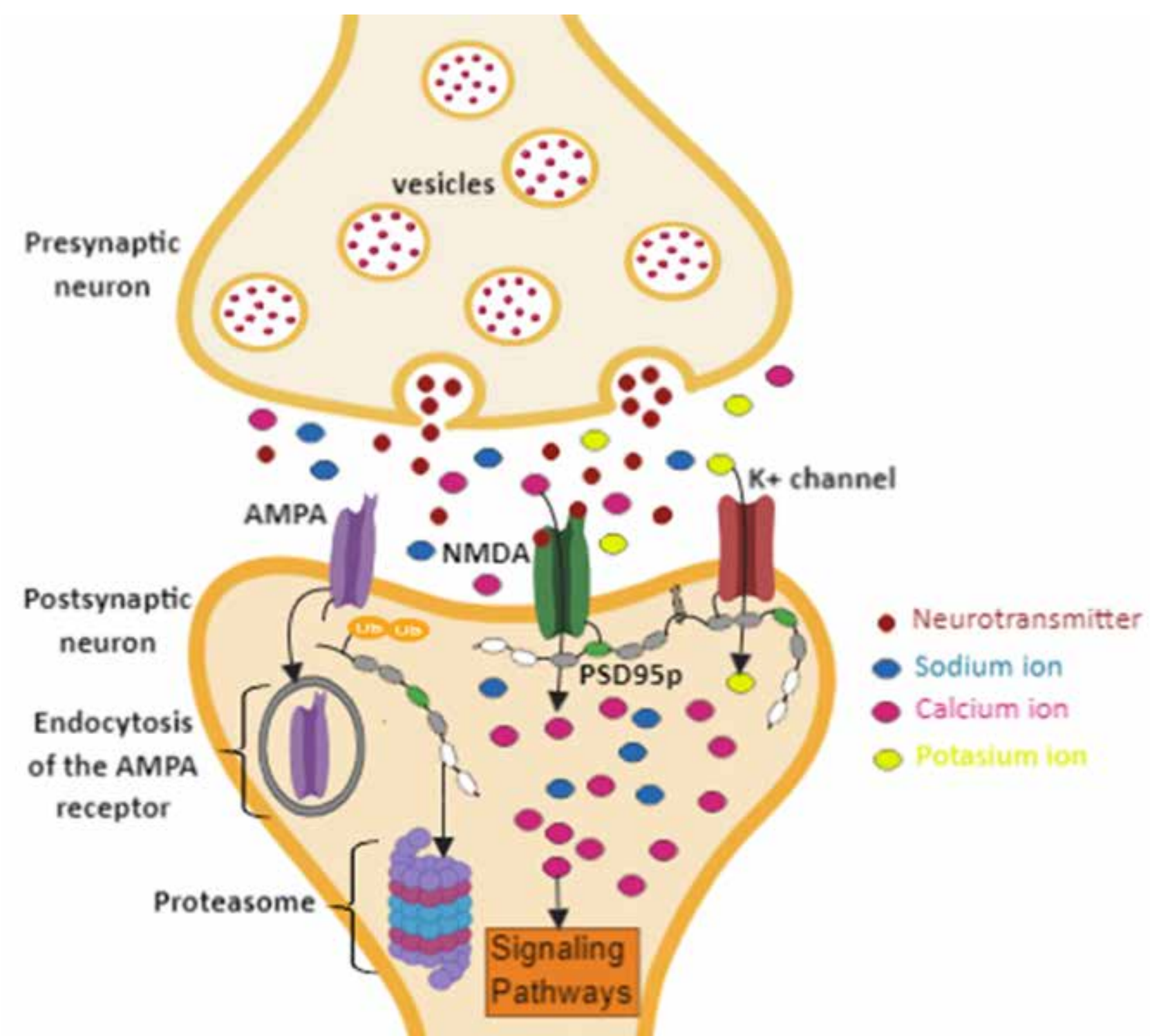

Figure 6.Regulation of synaptic strength by polyubiquitination of PSD95p. The polyubiquitination at the N-terminal end of PSD5p and subsequent proteolysis by the proteasome induces the delocalization and endocytosis of the AMPA receptors. This mechanism will decrease intracellularCa+ concentrations, modulating the synaptic connection between neurons that follow different activity profiles.

11. Hublin, J. J. The modern human colonization of western Eurasia: When and where? Quaternary Science Reviews 118, 194-210 (2015).

12. Quach, H. et al. Genetic Adaptation and Neandertal Admixture Shaped the Immune System of Human Populations. Cell 167, 643-656.e17 (2016).

13. Smith, F. H., Janković, I. \& Karavanić, I. The assimilation model, modern human origins in Europe, and the extinction of Neandertals. Quat. Int. 137, 7-19 (2005).

14. Falk, D. et al. Early hominid brain evolution: A new look at old endocasts. J. Hum. Evol. 38, 695-717 (2000)

15. Gunz, P., Neubauer, S., Maureille, B. \& Hublin, J. J. Brain development after birth differs between Neanderthals and modern humans. Current Biology 20, (2010).

16. Leigh, S. R. Brain growth, life history, and cognition in primate and human evolution. Am. J. Primatol. 62, 139-164 (2004).

17. Barton, R. A. \& Venditti, C. Rapid evolution of the cerebellum in humans and other great apes. Curr. Biol. 24, 2440-2444 (2014).

18. Marchetto, M. C. et al. Species-specific maturation profiles of human, chimpanzee and bonobo neural cells. Elife 8, (2019).

19. Carlson, K. J. et al. The endocast of MH1, Australopithecus sediba. Science (80-. ). 333, 1402-1407 (2011).
20.Banks, W. E. et al. Neanderthal extinction by competitive exclusion. PLoS One 3, (2008).

21. Hublin, J. J. The origin of Neandertals. Proceedings of the National Academy of Sciences of the United States of America 106, 16022-16027 (2009).

22. Wißing, C. et al. Stable isotopes reveal patterns of diet and mobility in the last Neandertals and first modern humans in Europe. Sci. Rep. 9, (2019)

23. Ponce de León, M. S., Bienvenu, T., Akazawa, T. \& Zollikofer, C. P. E. Brain development is similar in Neanderthals and modern humans. Current Biology 26, R665-R666 (2016).

24.Gunz, P. et al. Neandertal Introgression Sheds Light on Modern Human Endocranial Globularity. Curr. Biol. 29, 120-127.e5 (2019).

25. Stuchlik, A. Dynamic learning and memory, synaptic plasticity and neurogenesis: An update. Frontiers in Behavioral Neuroscience $8,(2014)$

26. Rogerson, T. et al. Synaptic tagging during memory allocation. Nature Reviews Neuroscience 15, 157-169 (2014).

27. Hsieh, J. Orchestrating transcriptional control of adult neurogenesis. Genes and Development 26, 1010-1021 (2012).

28. Boeckers, T. M. The postsynaptic density. Cell and Tissue Research 326, 409-422 (2006). 
29. Gray, N. W., Weimer, R. M., Bureau, I. \& Svoboda, K. Rapid redistribution of synaptic PSD-95 in the neocortex in vivo. PLoS Biol. 4, 2065-2075 (2006).

30.Kornau, H. C., Seeburg, P. H. \& Kennedy, M. B. Interaction of ion channels and receptors with PDZ domain proteins. Curr. Opin. Neurobiol. 7, 368-373 (1997).

31. Stathakis, D. G., Hoover, K. B., You, Z. \& Bryant, P. J. Human postsynaptic density-95 (PSD95): Location of the gene (DLG4) and possible function in nonneural as well as in neural tissues. Genomics 44, 71-82 (1997).

32. Carlisle, H. J., Fink, A. E., Grant, S. G. N. \& O'dell, T. J. Opposing effects of PSD-93 and PSD-95 on long-term potentiation and spike timing-dependent plasticity. J. Physiol. 586, 5885-5900 (2008).

33. Fernández, E. et al. Arc Requires PSD95 for Assembly into Postsynaptic Complexes Involved with Neural Dysfunction and Intelligence. Cell Rep. 21, 679-691 (2017).

34. Winkler, D. et al. Hypersocial behavior and biological redundancy in mice with reduced expression of PSD95 or PSD93. Behav. Brain Res. 352, 35-45 (2018).

35. Sheng, M. \& Sala, C. PDZ domains and the organization of supramolecular complexes. Annual Review of Neuroscience 24, 1-29 (2001).

36.Zhu, Y. C. et al. Palmitoylation-dependent CDKL5-PSD-95 interaction regulates synaptic targeting of CDKL5 and dendritic spine development. Proc. Natl. Acad. Sci. U. S. A. 110, 9118-9123 (2013).

37. Frank, R. A. \& Grant, S. G. Supramolecular organization of NMDA receptors and the postsynaptic density. Current Opinion in Neurobiology 45, 139-147 (2017).

38. Mir, S., Sen, T. \& Sen, N. Cytokine-Induced GAPDH Sulfhydration Affects PSD95 Degradation and Memory. Mol. Cell 56, 786-795 (2014).

39. Toro, C. \& Deakin, J. F. W. NMDA receptor subunit NRI and postsynaptic protein PSD-95 in hippocampus and orbitofrontal cortex in schizophrenia and mood disorder. Schizophr. Res. 80, 323-330 (2005).

40.Xing, J. et al. Resequencing and association analysis of Six PSD-95-related genes as possible susceptibility genes for schizophrenia and autism spectrum disorders. Sci. Rep. 6, (2016).

41. Ensembl Database. Available at: https://www.ensembl.org/index. html.

42.GenBank Database. Available at: https://www.ncbi.nlm.nih.gov/ genbank/.

43. Neanderthal Genome database.

44. MEGA X software. Available at: https://www.megasoftware.net/.

45.Phyre 2 online software. Available at: http://www.sbg.bio.ic.ac.uk/ phyre2/html/page.cgi?id=index.

46.SWISS-MODEL online software. Available at: https://swissmodel. expasy.org/interactive.

47. PyMOL software. Available at: https://pymol.org/2/.

48.Kumar, S., Stecher, G., Li, M., Knyaz, C. \& Tamura, K. MEGA X: Molecular evolutionary genetics analysis across computing platforms. Mol. Biol. Evol. 35, 1547-1549 (2018).

49. Tamura, K. \& Nei, M. Estimation of the number of nucleotide substitutions in the control region of mitochondrial DNA in humans and chimpanzees. Mol. Biol. Evol. 10, 512-526 (1993).

50.Le, S. Q. \& Gascuel, O. An improved general amino acid replacement matrix. Mol. Biol. Evol. 25, 1307-1320 (2008)

51. Felsenstein, J. Confidence Limits on Phylogenies: An Approach Using the Bootstrap. Evolution (N. Y). 39, 783 (1985).

52. Colledge, M. et al. Ubiquitination regulates PSD-95 degradation and AMPA receptor surface expression. Neuron 40, 595-607 (2003).

53. Xu, W. et al. Molecular Dissociation of the Role of PSD-95 in Regulating Synaptic Strength and LTD. Neuron 57, 248-262 (2008).

54. Topinka, J. R. \& Bredt, D. S. N-terminal palmitoylation of PSD-95 regulates association with cell membranes and interaction with K+ channel K(v)1.4. Neuron 20, 125-134 (1998).

55.Fukata, M., Fukata, Y., Adesnik, H., Nicoll, R. A. \& Bredt, D. S. Identification of PSD-95 palmitoylating enzymes. Neuron 44 987-996 (2004).
56. Byrne, J. H., Heidelberger, R., Waxham, M. N. \& Roberts, J. L. From Molecules to Networks. From Molecules to Networks (2003). doi:10.1016/B978-0-12-148660-0.X5000-8

57. Globa, A. K. \& Bamji, S. X. Protein palmitoylation in the development and plasticity of neuronal connections. Current Opinion in Neurobiology 45, 210-220 (2017).

58. Purkey, A. M. et al. AKAP150 Palmitoylation Regulates Synaptic Incorporation of Ca2+-Permeable AMPA Receptors to Control LTP. Cell Rep. 25, 974-987.e4 (2018).

59.Flores-Soto, M. E. et al. Estructura y función de las subunidades del receptor a glutamato tipo NMDA. Neurologia 27, 301-310 (2012)

60.Zhang, Y. et al. Capping of the N-terminus of PSD-95 by calmodulin triggers its postsynaptic release. EMBO J. 33, 1341-1353 (2014).

61. Jaffrey, S. R., Snowman, A. M., Eliasson, M. J. L., Cohen, N. A. \& Snyder, S. H. CAPON: A protein associated with neuronal nitric oxide synthase that regulates its interactions with PSD95. Neuron 20, 115-124 (1998).

62. Smith, A. E. et al. Source memory in rats is impaired by an NMDA receptor antagonist but not by PSD95-nNOS protein-protein interaction inhibitors. Behav. Brain Res. 305, 23-29 (2016).

Received: 17 november 2020

Accepted: 15 january 2021 